\title{
Autopsia por silico-asbestosis. Revisión a propósito de un caso forense
}

\section{Autopsy by silico-asbestosis. A review of a forensic case}

\begin{abstract}
Resumen
Se presenta el caso de un varón de 72 años de edad, ex fumador desde hace años, diagnosticado de silicosis y carcinoma epidermoide de pulmón derecho, solicitándose por parte de la familia autopsia judicial por la sospecha de padecimiento de asbestosis y tras su confirmación reclamación como enfermedad profesional. La exposición de este suceso nos permite realizar una revisión de una enfermedad profesional que suele pasar como desconocida en las salas de autopsia, a pesar de su repercusión judicial. La asbestosis es un tipo de neumoconiosis reconocida como enfermedad profesional producida por la inhalación de fibras de asbesto. Su inhalación provoca fibrosis pulmonar. Presenta un tiempo de latencia clínica inversamente proporcional al nivel de exposición. Además de fibrosis pulmonar pueden producirse derrames pleurales, placas fibrosas pleurales, mesoteliomas, carcinomas pleurales y carcinoma de laringe. La silicosis es la neumoconiosis producida por inhalación de partículas de sílice, entendiendo por neumoconiosis la enfermedad ocasionada por el depósito de polvo en los pulmones con una reacción patológica frente a este, en especial de tipo fibroso. La silicosis es una enfermedad fibrótica-pulmonar de carácter irreversible y considerada enfermedad profesional incapacitante en muchos países, una de cuyas variantes más raras es la silico-asbestosis, la cual requiere exposición al sílice y al amianto.
\end{abstract}

Palabras clave: Asbestosis. Fibrosis pulmonar. Placas pleurales. Silico-asbestosis.

\begin{abstract}
The case is presented the case of a 72-year-old ex-smoker for years, diagnosed with silicosis and squamous cell carcinoma of the right lung, being requested by the judicial autopsy family suspected of suffering from asbestosis and after confirmation complaint as an occupational disease. The exposure of this event allows us to conduct a review of an occupational disease usually happens as unknown in autopsy rooms despite its legal repercussions. Asbestosis is a type of pneumoconiosis recognized as an occupational disease caused by inhaling asbestos fibers. Inhalation causes pulmonary fibrosis in the lung presenting a time inversely proportional to the exposure level clinical latency. Besides pulmonary fibrosis may occur pleural effusions, pleural fibrous plaques, mesothelioma, pleural carcinomas and laryngeal carcinoma. Silicosis is produced by inhalation pneumoconiosis silica particles, meaning the disease caused by pneumoconiosis dust deposit in the lungs with a pathological reaction to the same, especially fibrous. Silicosis is a fibrotic lung disease-and considered irreversible disabling occupational disease in many countries one of which is the more rare variants silico asbestosis, which requires exposure to silica and asbestos.
\end{abstract}

Key words: Asbestosis. Pulmonary fibrosis. Pleural plaques. Silico asbestosis.

\section{A. Sibón Olano ${ }^{1}$ \\ E. Sánchez Rodríguez ${ }^{1}$ \\ E. Barrera Pérez ${ }^{1}$ \\ J. Larrondo Espinosa ${ }^{2}$ \\ M. Salguero Villadiego ${ }^{3}$}

${ }^{1}$ Médico Forense, Servicio de Patología, IML de Cádiz ${ }^{2}$ Facultativo Histopatología, INTCF, Sevilla

${ }^{3}$ Jefe de Servicio de Histopatología, INTCF, Sevilla.

Correspondencia:

A. Sibón Olano

Servicio de Patología Forense del IMLCA

$\mathrm{C} /$ Chiclana esquina con

Algodonales, $s / n$

Tanatorio SERVISA

11001 Cádiz

E-mail:

agustin.sibon.ius@juntadeandalucia.es
Fecha de recepción: 10. NOV. 2014

Fecha de aceptación: 09. DIC. 2014 


\section{Introducción}

Neumoconiosis es un término que se acuñó para describir la reacción pulmonar no neoplásica a la inhalación de polvos minerales, en la gran mayoría de los casos secundaria a exposiciones laborales ${ }^{1}$. Existe una relación directa entre la intensidad y la duración de la exposición y la cantidad de polvo depositado en el pulmón, siendo característico un tiempo de latencia prolongado entre la exposición al agente causal y la aparición de la sintomatología o el diagnóstico de la enfermedad ${ }^{2}$. Se caracteriza por una patología pulmonar intersticial difusa que presenta manifestaciones clínicas, radiológicas y funcionales respiratorias comunes, que afectan a la estructura alvéolo-intersticial, a la pequeña vía aérea y a la vasculatura pulmonar ${ }^{3}$.

Dentro de las neumoconiosis, la asbestosis es una enfermedad pulmonar causada por la inhalación de asbesto. Los asbestos son silicatos hidratados de magnesio que han sido utilizados en diferentes ocupaciones e industrias por sus cualidades de ser flexibles y resistentes a la tensión, a la descomposición térmica y a diferentes sustancias químicas. Generan fibrosis pulmonar difusa y progresiva que dará lugar a insuficiencia respiratoria, con una alta morbilidad y discapacidad como enfermedad ocupacional, ya que no tiene cura, es una patología irreversible y no existe tratamiento ${ }^{4}$.

Por otro lado, en la silicosis son las partículas respirables de sílice (menores de $5 \mu$ ) la que alcanzan el parénquima pulmonar y quedan retenidas para ser fagocitadas por los macrófagos pasando a sus lisosomas, pero los mecanismos destructivos de que disponen estos son inútiles frente a la sílice; el macrófago acaba destruido y libera en el medio enzimas y radicales que potencian la inflamación y generan más radicales oxidantes y enzimas que no son capaces de destruir la sílice, pero sí de lesionar el propio tejido pulmonar, conduciendo a la fibrosis ${ }^{5}$. Es por esto que se ha propuesto una hipótesis inflamatoria en la base de la patogenia de la silicosis. Los sujetos que no controlen bien la respuesta inflamatoria podrían estar en desventaja 6 .

Estos materiales se utilizan en construcción, minería, manufactura de plásticos y cauchos, reparación naval y de camiones, ferrocarriles, electricidad, soldadura, etc. ${ }^{4}$.

Las dos principales fibras de asbesto son:

- Forma serpentina, representada por el crisolito.

- Forma anfibólica, representada por la amosita, la crocidolita, la antofilita, la tremolita y la actinolita.

La sílice cristalina o dióxido de silicio $\left(\mathrm{SiO}_{2}\right)$ es la que ocasiona la silicosis. Se encuentra en la naturaleza en forma de cuarzo, cristobalita o tridimita, siendo el cuarzo el más abundante ( $12 \%$ de la corteza terrestre); de ahí que la exposición a sílice sea muy frecuente ${ }^{7}$.

En el asbesto, los tipos presentan diferentes estructuras y propiedades físico-químicas. Las fibras de crisolito son onduladas y forman haces, mientras que las de tipo anfibólico son más largas que las primeras y tienen forma de aguja. Estas diferencias son la causa de los diversos usos del asbesto y también, en parte, de su capacidad patogénica, siendo el crisolito, la amosita y la crocidolita los implicados en la gran mayoría de las enfermedades pleuropulmonares por asbesto $^{1}$.

\section{Etiopatogenia}

Por las características mencionadas anteriormente, tanto la silicosis como la asbestosis fueron reguladas por Orden de 12 de enero de 1963, por la que se aprobaron las normas reglamentarias de carácter médico por las que se rigieron el reconocimiento, el diagnóstico y la calificación de las enfermedades profesionales, quedando recogidas como enfermedades causadas por agentes animados y definiéndose los cuadros clínicos con derecho a reparación por el Seguro, las normas para el reconocimiento previo al ingreso en labores con riesgo profesional asbestósico, así como las normas para los reconocimientos periódicos y para el diagnóstico de la asbestosis, y para la calificación de la capacidad ${ }^{8}$.

La silicosis fue la primera enfermedad profesional que se reguló en España por la Ley Dato de 1900, que pasó a regular el accidente de trabajo creando el Seguro para el mismo y adoptando frente a la doctrina de la culpa hasta entonces imperante, la doctrina del riesgo profesional, y estableció que los empresarios debían proteger a sus trabajadores frente a los riesgos en el trabajo y sus consecuencias. Actualmente esta enfermedad se encuentra regulada como enfermedad profesional en el Real Decreto 1299/2006 de 10 de noviembre, dentro del cuadro de enfermedades profesionales en el Sistema de la Seguridad Social ${ }^{9}$.

Por resolución del Parlamento Europeo de 1978, el asbesto se declaró como sustancia cancerígena. Las variedades de asbesto azul y marrón fueron prohibidas en España en 1984 y 1993. En 1999 
se prohibió la comercialización y el uso de cualquier tipo de asbesto. En diciembre de 2001 se prohibió la comercialización y utilización de asbesto blanco mediante la Orden Ministerial de 7 de diciembre de 2001. El 15 de junio de 2002 entró en vigor en España la prohibición de fabricar productos que contengan asbesto. Hasta el 15 de diciembre de 2002 podían comercializarse e instalarse productos con asbesto fabricados antes del 15 de junio de ese año ${ }^{10}$.

La legislación española sobre el asbesto está desarrollada en el Real Decreto 396/2006 de 31 de marzo, estableciéndose las disposiciones mínimas de seguridad y salud aplicables a los trabajos con riesgo de exposición al asbesto ${ }^{10}$.

En relación al tipo de exposición se diferencian:

- La exposición ocupacional (que es la principal), en la cual las fibras de sílice y asbesto pueden liberarse al aire por manipulación de los materiales que los contienen, principalmente oficios de minería de chorro de arena y roca dura en el caso de la silicosis.

- La exposición doméstica, en la cual las partículas llegan por vía indirecta y se da en familiares de trabajadores cuando entran en contacto con fibras y polvo de sílice que quedan en la ropa de trabajo.

- La exposición ambiental, que afecta a personas que residen cerca de un punto de emisión de asbesto o sílice e inhalan el polvo disperso en el aire ${ }^{10}$.

Intentaremos profundizar en el mundo de la patología del asbesto, y haremos un estudio más somero de la patología del sílice, por considerar que la asbestosis no solo es una neumoconiosis más desconocida para el mundo médico forense, sino que además hacerlo de este modo nos permite un estudio individualizado de la silicosis en otra ocasión, con la exposición de un nuevo caso.

El riesgo de que se presente una enfermedad asociada al asbesto está relacionado con la concentración de fibras presente en el aire, la duración y la frecuencia de la exposición, el tamaño de las fibras y el tiempo transcurrido desde la exposición inicial ${ }^{10}$.

Muchos estudios han informado de la asociación entre la presencia de enfermedad benigna pleural y asbestosis y el aumento del riesgo de mesotelioma maligno. Koskinen et al. ${ }^{11}$ encontraron que las personas expuestas al asbesto con evidencia radiográfica de placas pleurales tenían un reducido riesgo de mesotelioma en comparación con aquellas personas que no presentaban placas pleurales. Sin embargo, su estudio no tenía en cuenta el grado, la duración ni el tipo de la exposición, y tampoco el tiempo desde esta, índices que se sabe que son determinantes del riesgo de mesotelioma ${ }^{12}$.

La asbestosis produce fibrosis por interacción con los macrófagos pulmonares, pero no está del todo clara la razón por la que algunos polvos inorgánicos causan fibrosis nodular y otros, como el amianto, originan fibrosis intersticial difusa ${ }^{1}$.

Es conocida la relación entre el consumo de cigarrillos y las enfermedades por el asbesto, si bien el mecanismo de la interacción de ambos permanece inexplicado. Tanto los fumadores como los ex fumadores presentan una mayor frecuencia de alteraciones radiográficas relacionadas con el asbesto que la población general, y la mortalidad debida a enfermedades pulmonares por el asbesto es mayor entre los trabajadores expuestos que fuman que entre aquellos que no lo hacen ${ }^{13}$.

Según su tamaño, las fibras de asbesto van depositándose por impactación, sedimentación o intercepción en las bifurcaciones bronquiales, los bronquiolos respiratorios y los alvéolos, desde donde migran al intersticio. Esto da lugar a una acumulación de macrófagos en los conductos alveolares, el intersticio peribronquiolar y los espacios alveolares, provocando una alveolitis macrofágica ${ }^{14}$.

Estas células tienden a fagocitar las fibras que serán finalmente eliminadas a través de la corriente mucociliar. Si la cantidad de fibras es tan importante que supera la capacidad fagocítica de los macrófagos, su aclaramiento no llega a ser total. Las fibras de asbesto no pueden ser fagocitadas de manera completa y uniforme, y estimulan la producción de diversas sustancias por parte de los macrófagos. Dichas sustancias incluyen radicales libres de oxígeno, que provocan lesión celular directa y peroxidación de las membranas celulares, activadores del plasminógeno, fibronectina y diversas citocinas que contribuyen a la fibrosis tisular estimulando la proliferación y la quimiotaxis de los fibroblastos y la síntesis de colágeno ${ }^{15}$.

La exposición crónica hace que se desarrolle dentro del pulmón una reacción inflamatoria crónica con infiltración de múltiples células, que progresará de forma lenta a fibrosis pulmonar. El grado de fibrosis es dependiente de la cantidad de asbesto a la que se expuso el individuo ${ }^{4}$.

Las partículas minerales se inhalan habitualmente en forma de mezclas de sílice y amianto, aunque uno de los componentes puede predominar y determinar la respuesta. Las lesiones a menudo poseen una 
estructura característica, de acuerdo con el principal tipo de partícula depositada ${ }^{16}$.

La lesión más precoz es la fibrosis de las paredes de los bronquiolos respiratorios, con engrosamiento de los septos adyacentes. Asimismo se produce una intensa reacción celular peribronquiolar que puede estenosar u obstruir la luz bronquiolar. En el intersticio se acumulan linfocitos y puede haber proliferación de fibras musculares lisas. Progresivamente se afectan los bronquiolos de primer, segundo y tercer orden, y la fibrosis se va haciendo difusa. Se produce una remodelación de la arquitectura pulmonar que conduce al pulmón en "panal de abeja", sin que, a diferencia de otras neumoconiosis, aumente el tamaño de los ganglios linfáticos ni se produzca una fibrosis masiva progresiva $^{17}$.

Patológicamente, la asbestosis es indistinguible de otras enfermedades difusas del parénquima pulmonar salvo por la presencia de los cuerpos de asbesto y de fibras de asbesto sin revestimiento. Los cuerpos de asbesto, visibles a menudo en las preparaciones histológicas, consisten en los denominados "corpúsculos de amianto" que se observan como bastoncillos fusiformes o arrosariados, de color marrón dorado con un centro translúcido (por lo que algunos lo denominan invisible). A gran aumento se observa un núcleo compuesto por una fibra invisible o translúcida de asbesto (habitualmente amosita o crocidolita) de 20 a $50 \mu \mathrm{m}$ de longitud, rodeado por un revestimiento continuo o discontinuo de hierro y proteínas de grosor variable entre 2 y $5 \mu \mathrm{m}$. Su morfología es variable, dependiendo de la longitud del núcleo de asbesto y del patrón de disposición del hierro y las proteínas; puede adoptar una forma característica de "palillo de tambor"17. Parecen formarse cuando los macrófagos intentan fagocitar las fibras de amianto.

El patrón de la silicosis es el nódulo silicótico que macroscópicamente, en sus estadios iniciales, se identifica como un nódulo minúsculo bien delimitado y de diverso color, de pálido a negruzco. Microscópicamente se reconoce por fibras de colágeno hialinizadas dispuestas concéntricamente alrededor de un centro amorfo con el típico "aspecto en remolino"18.

La exposición profesional al amianto puede originar:

- Fibrosis intersticial parenquimatosa (asbestosis).

- Carcinoma broncógeno.

- Derrames pleurales.

- Placas fibrosas localizadas o, raras veces, fibrosis pleural difusa.

- Mesoteliomas pleurales y peritoneales.

- Carcinoma de laringe ${ }^{1}$.
La distribución de las fibras en la pleura ocurre sobre todo en la hoja parietal, pero de forma irregular, agrupadas en focos, siendo las placas pleurales lesiones fibrosas, que constituyen la patología pleural más precoz después del derrame pleural benigno. En general son asintomáticas aunque sean relativamente extensas, encontrándose circunscritas y formadas por tejido fibroso en la pleura parietal, múltiples y localizadas de manera preferente en las zonas intercostales laterales y posteriores, en la pleura mediastínica y en la cúpula diafragmática. Suelen ser bilaterales, pero no simétricas, y calcifican con frecuencia. Se consideran un marcador de la carga de exposición y su presencia puede aumentar el riesgo de padecer tanto asbestosis como mesotelioma, no por degeneración maligna de la placa sino por el riesgo asociado a la carga de asbesto ${ }^{19}$.

\section{Clínica}

Las manifestaciones clínicas de la silicosis y la asbestosis son indistinguibles de las de cualquier otra neumopatía intersticial difusa. Es típica la aparición de disnea entre 10 y 20 años después de la exposición, que suele ir acompañada de tos productiva. La enfermedad puede permanecer estática o progresar hacia insuficiencia cardíaca congestiva, cor pulmonale y la muerte. El desarrollo del síndrome de Caplan (inflamación y cicatrización pulmonar a consecuencia de la inhalación de polvo en los trabajadores de minas de carbón) acelera la evolución clínica. Las placas pleurales, casi siempre asintomáticas, se detectan en las radiografías como densidades circunscritas. El cáncer de pulmón o pleural relacionado con la exposición al amianto tiene un pronóstico especialmente sombrío ${ }^{1}$.

\section{Diagnóstico}

La radiografía simple de tórax presenta, como principal hallazgo en la asbestosis, placas pleurales que son engrosamientos o calcificaciones a lo largo de la pleura parietal, predominantes en la parte inferior del pulmón, en el diafragma y en el reborde cardiaco ${ }^{4}$.

En la silicosis se observa radiográficamente un patrón fino nodular en los campos pulmonares superiores, y también es posible su asociación a tuberculosis ${ }^{18}$.

Actualmente, una radiografía de tórax anormal presenta un valor predictivo positivo bajo en las personas expuestas a trabajos relacionados con el asbesto. El 
diagnóstico puede establecerse por las manifestaciones clínicas, la presencia de placas pleurales o fibrosis intersticial pulmonar, y la reducción de la capacidad de difusión del monóxido de carbono 20 .

La tomografía computarizada (TC) de alta resolución, por su alto coste, solo está indicada cuando la radiografía de tórax no es concluyente 4 . Sólo el $50 \%$ al $80 \%$ de las placas vistas en autopsia, TC convencional o TC de alta resolución son identificadas también en la radiografía ${ }^{21}$. Diego et al..$^{22}$ estudiaron muestras pulmonares obtenidas de las autopsias de 30 varones, con una edad media de 67 años, trabajadores de los astilleros de Ferrol, y encontraron un aumento de los niveles de amianto en los pulmones.

En el caso que nos ocupa, el diagnóstico de silicosis se encontraba efectuado y reconocido a efectos de incapacidad laboral. Se trataba, pues, de llegar a un diagnóstico post mórtem de asbestosis mediante una autopsia médico-forense para evidenciar una silico-asbestosis.

Macroscópicamente, el examen de la cavidad torácica permitió objetivar un aumento de tamaño y de peso de ambos pulmones (pulmón derecho 878 gramos y pulmón izquierdo 580 gramos), congestivos, con depósitos antracóticos numerosos en su superficie (Figura 1). El pulmón derecho presentaba numerosas adherencias a la pared costal, con engrosamiento, debido a la existencia de placas duras de color blancoparduzco, semejantes a gotas de cera fundidas, al igual que la pared costal derecha (en forma de placas arrosariadas) (Figuras 2 y 3 ). Además, dicho pulmón mostraba formaciones tumorales duras diseminadas y numerosos ganglios afectados, junto con fibrosis pulmonar. En las caras anteriores y posteriores torácicas, así como en las cúpulas diafragmáticas, había grandes placas de color blanco-parduzco (Figura 4). Asimismo, el pericardio presentaba granos de color blanco-parduzco en su cara basal. En el corazón encontramos hipertrofia ventricular izquierda (grosor del miocardio $2,1 \mathrm{~cm}$ ) y arteriosclerosis coronaria moderada.

En otros casos similares en los que hemos tenido que actuar, durante la autopsia no se ha encontrado fibrosis difusa del pulmón, pero sí un engrosamiento pleural difuso junto con grandes cantidades de cuerpos de asbesto en el tejido pulmonar en el estudio histológico.

Lo anterior, junto con una historia laboral detallada recogida en el acto del levantamiento del cadáver, constituye una información útil para la determinación de la causa del fallecimiento ${ }^{23}$.
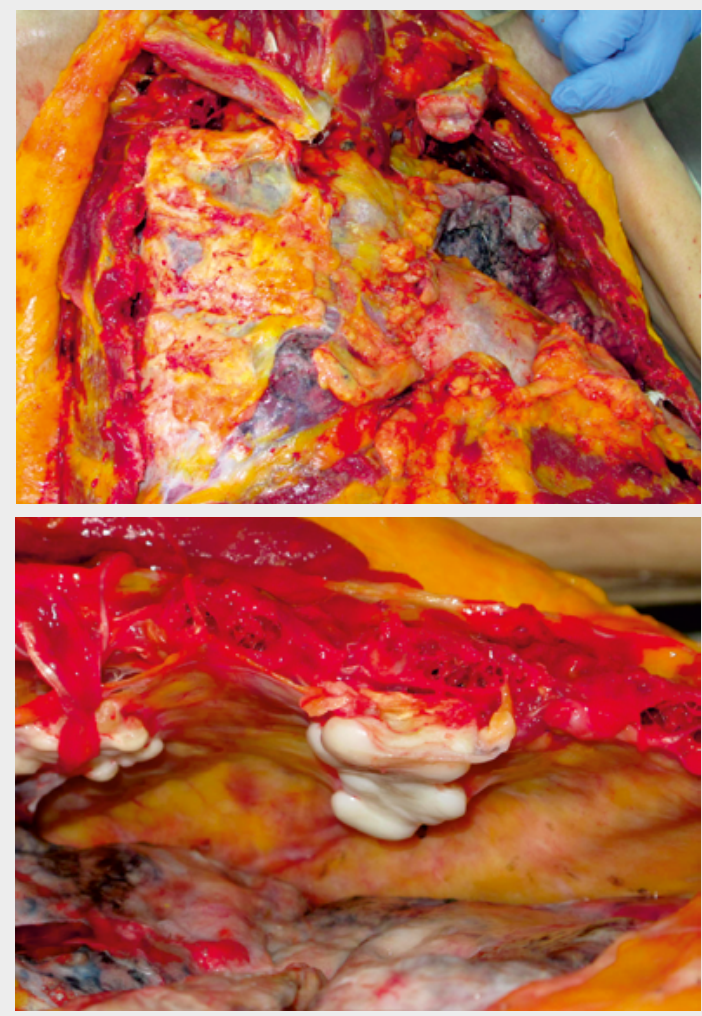

Figura 2.

Placas de color

blanco-parduzco en la pleura intercostal derecha. Morfología "en gota de cera".
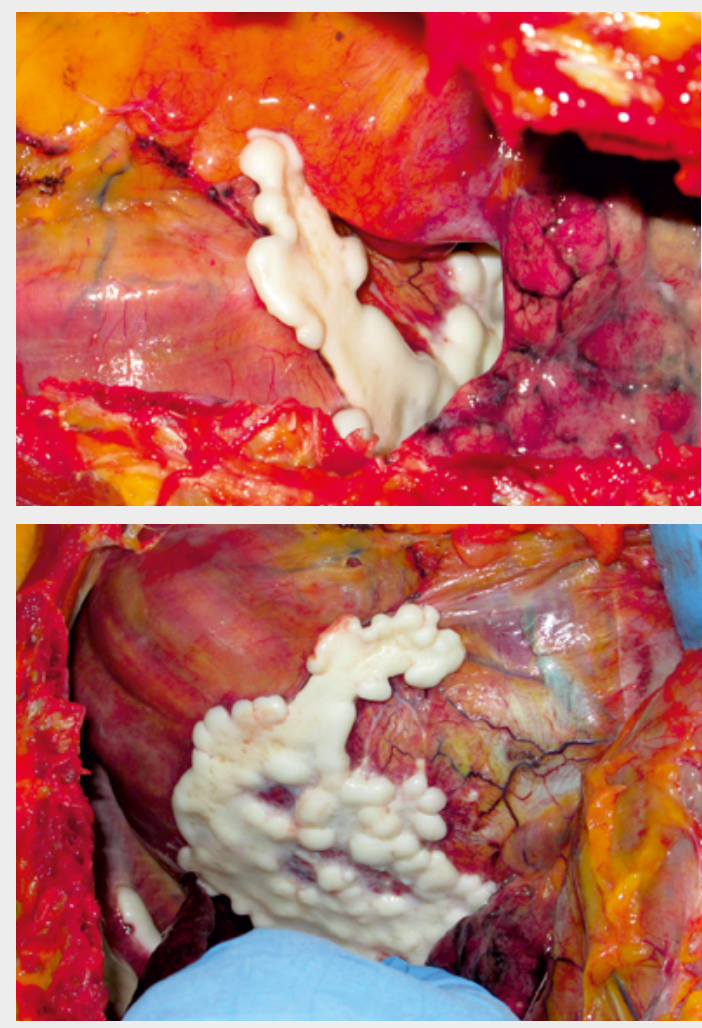

Figura 3.

Placas de color

blanco-parduzco en la cúpula diafragmática. Morfología "en gota de cera".

Figura 4.

Placas de color

blanco-parduzco en la cúpula diafragmática. Morfología "en gota de cera". 
Figura 5. Placa pleural de asbestosis.

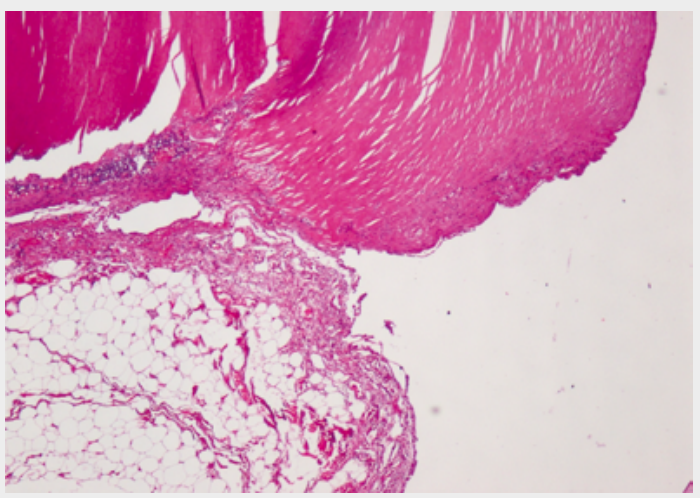

Figura 6. Nódulo silicótico.

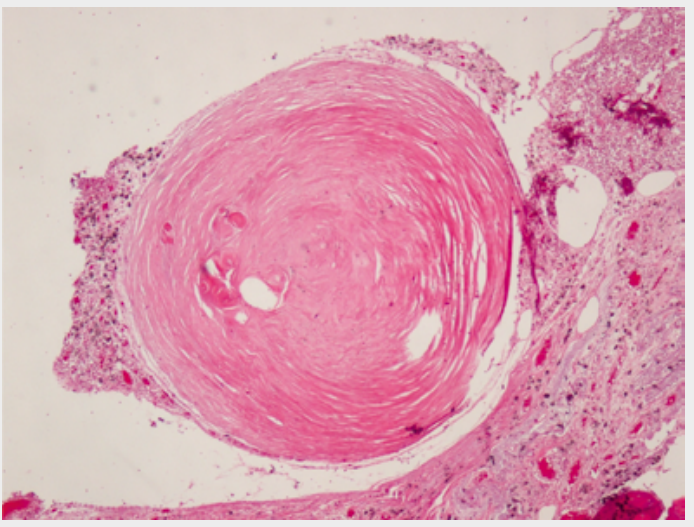

Figura 7.

Nódulo silicótico en remolino.

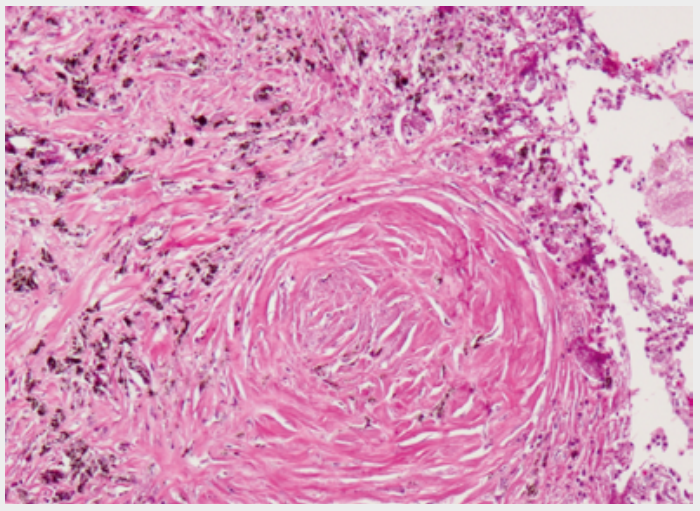

Figura 8.

Cuerpo de asbesto "en palillo de tambor".

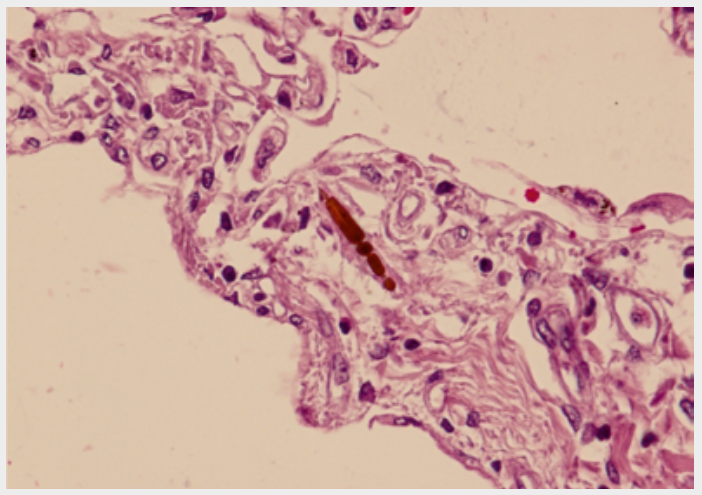

En este caso que presentamos, microscópicamente se encontraron en la pleura parietal, en su pared interna, grandes placas bien circunscritas de aspecto densamente fibroso y de color blanco brillante, con el diagnóstico histopatológico confirmado de placas pleurales de asbestosis (Figura 5), mientras que en los pulmones se observan congestión, amplias áreas con espacios alveolares ocupados por polimorfonucleares muy numerosos junto con focos con hematíes, descamación de macrófagos y depósitos dispersos de pigmento antracótico, rotura de septos con formación de bullas de distribución difusa y fibrosis intersticial, y lesiones nodulares pequeñas que se componen de capas concéntricas de colágeno hialinizado rodeadas por una cápsula densa de colágeno más condensado que presentan también pigmento antracótico.

El diagnóstico histopatológico de las muestras de pulmón remitidas consistió en carcinoma pobremente diferenciado, nódulos silicóticos (Figuras 6 y 7), bronconeumonía aguda grave, congestión, edema, hemorragia intraalveolar aguda focal, cambios de enfisema bulloso y antracosis. En definitiva, pudo concluirse la confirmación diagnóstica de la coexistencia de carcinoma con silicosis, como era conocido, así como la evidencia macroscópica y microscópica de asbestosis (Figuras 5 y 8), lo que posibilitó una reclamación de enfermedad profesional por la familia del fallecido.

\section{Tratamiento}

No existe ningún tratamiento eficaz para la silicosis ni para la asbestosis, como tampoco para la silicoasbestosis. Las únicas medidas eficaces son las preventivas $^{24}$.

\section{Conclusiones}

A pesar de la normativa vigente sobre el uso del amianto y de que laboralmente se utilizan todas las medidas de protección adecuadas para impedir su inhalación, los casos de adquisición de la enfermedad que encontramos en el ámbito forense se remontan a décadas atrás y son consecuencia del desconocimiento de las consecuencias de la falta de prevención.

El cuadro característico que nos encontramos corresponde a un varón mayor de 70 años con insuficiencia respiratoria de evolución y antecedentes de trabajo relacionado con el amianto. Macroscópicamente, en la autopsia se observan las placas características de 
la asbestosis, de color blanco-parduzco y duras al tacto, con morfología "en gota de cera", en la pleura diafragmática e incluso en las paredes de pericardio, que son patognomónicas de la enfermedad, mientras que microscópicamente se observa fibrosis pulmonar junto con la presencia de pigmentos férricos con disposición "en palillo de tambor".

No es inusual la combinación con la silicosis, formando una silico-asbestosis que requiere la exposición a los dos patógenos en las industrias de riesgo. En este caso, la enfermedad es mucho más incapacitante para quien la padece, y junto al anterior patrón encontremos que macroscópicamente no es tan espectacular como la asbestosis, pero al microscopio los nódulos "en forma de remolino" mostrarán el patrón típico de esta.
El informe del levantamiento del cadáver que recoja los antecedentes laborales del fallecido, junto con los hallazgos macroscópicos referidos, deberán hacer sospechar al patólogo forense la existencia de asbestosis, la cual será confirmada con los estudios histopatológicos. Además, en ausencia de identificación del fallecido, la existencia de placas pleurales macroscópicas de estas características contribuye a su filiación entre las diversas posibilidades y candidatos, al permitir ubicarlo en un determinado tipo de industria y, por ende, en su localización geográfica.

Los autores declaran no tener ningún conflicto de intereses.

\section{Bibliografía}

1. Maitra A. Enfermedades ambientales. En: Kumar V, Cotran RS, Robbins SL, editores. Patología humana. $7^{a}$ ed. Madrid: Elsevier; 2004. p. 265-306.

2. Bermúdez JA, de Miguel Díez J. Enfermedades pulmonares intersticiales difusas. Madrid: Ergón; 2008.

3. Xaubet A, Ancochea J, Blanquer R, Montero C, Morell $F$, Rodríguez Becerra $\mathrm{E}$, et al. Diagnóstico y tratamiento de las enfermedades pulmonares intersticiales difusas. Arch Bronconeumol. 2003; 39(12):580-600.

4. Agudelo SPE, de la Cuesta Herrera S, Correa NG, Ramírez MCG, Montoya JM, Zapata SR, et al. Asbestosis: epidemiología, prevención y tratamiento. Revista CES Salud Pública. 2012;3(2):251-8.

5. Mossman BT, Churg A. Mechanisms in the pathogenesis of asbestosis and silicosis. Am J Respir Crit Care Med. 1998;157(5):1666-80.

6. Fernández RR. Enfermedades respiratorias ocupacionales y medioambientales: fundamentos para su investigación clínico-epidemiológica. Sociedad Asturiana de Medicina y Seguridad del Trabajo; 2011.

7. Martellosio V, Scafa F, Colombi R, Candura SM Silicoasbestosi: presentazione di tre casi clinici. $G$ Ital Med Lav Erg. 2007;29(3):735-6.

8. Boletín Oficial del Estado. (Consultado el 29/10/2013.) Disponible en: www.boe.es/boe/ dias/1963/03/13/pdfs/A04218-04227.pdf

9. Boletín Oficial del Estado. Real Decreto por el que se aprueba el cuadro de enfermedades profesionales en el sistema de la Seguridad Social. BOE núm. 302 de 19-12-2006.

10. Luis G, Hernández C, Rubio C, Frías I, Gutiérrez A, Hardisson A. Toxicología del asbesto. Cuadernos de Medicina Forense. 2009(57):207-13.

11. Koskinen K, Pukkala E, Martikainen R, Reijula $\mathrm{K}$, Karjalainen A. Different measures of asbestos exposure in estimating risk of lung cancer and mesothelioma among construction workers. J Occup Environ Med. 2002;44(12):1190-6.

12. Reid A, De Klerk N, Ambrosini G, Olsen N, Pang S, Musk $A$. The additional risk of malignant mesothelioma in former workers and residents of Wittenoom with benign pleural disease or asbestosis. Occup Environ Med. 2005;62(10):665-9.

13. Lilis R, Selikoff IJ, Lerman Y, Seidman H, Gelb SK. Asbestosis: interstitial pulmonary fibrosis and pleural fibrosis in a cohort of asbestos insulation workers: influence of cigarette smoking. Am J Ind Med. 1986;10(5-6):459-70.

14. Rom WN, Bitterman PB, Rennard SI, Cantin A, Crystal RG. Characterization of the lower respiratory tract inflammation of nonsmoking individuals with interstitial lung disease associated with chronic inhalation of inorganic dusts. Am Rev Respir Dis. 1987;136(6):1429-34

15. Rom WN, Basset P, Fells GA, Nukiwa T, Trapnell BC, Crysal RG. Alveolar macrophages release an insulin-like growth factor I-type molecule. J Clin Invest. 1988;82(5):1685-93. 
16. Heppleston AG. Pulmonary toxicology of silica, coal and asbestos. Environ Health Perspect. 1984;55:111-27.

17. Churg AM, Warnock ML. Asbestos and other ferruginous bodies: their formation and clinical significance. Am J Pathol. 1981;102(3):447-56.

18. Maitra A, Kumar V. El pulmón y el aparato respiratorio superior. En: Kumar V, Abbas Abul K, Aster J, editores. Patología humana. $9^{\mathrm{a}}$ ed. Madrid: Elsevier; 2012. p. 453-508.

19. Boldú J, Eguía V. Enfermedades pleurales benignas inducidas por asbesto. An Sist Sanit Navar. 2005;28(Supl 1).

20. Ross RM. The clinical diagnosis of asbestosis in this century requires more than a chest radiograph. Chest. 2003;124(3):1120-8.
21. Smith D. Diagnosis and initial management of nonmalignant diseases related to asbestos. Am J Respir Crit Care Med. 2004;170(6):691-715.

22. Diego C, Velasco-García MI, Cruz MJ, Untoria MD Morell $F$, Ferrer J. Asbestos pulmonary content in workers of Ferrol shipyards, Spain. Med Clin (Barc). 2013;140(4):152-6.

23. Kinoshita H, Matsui K, Kubota A, Kasuda S, Nishiguchi M, Ouchi $\mathrm{H}$, et al. Pulmonary asbestosis: an autopsy case. Nihon Hoigaku Zasshi. 2007;61(2):12933.

24. Marín Martínez B, Clavera I. Asbestosis. An Sist Sanit Navar. [revista on line]. (Consultado el 4/12/2014.) Disponible en: http://scielo.isciii. es/scielo. php?script $=$ sci arttext\&pid=S1137 66272005000200006\&Ing=es. http://dx.doi. org/10.4321/S1137-66272005000200006. 